\title{
Electron- microscopy characterization of cells infected with a new phlebovirus isolated in sandflies from South Portugal
}

\author{
Amaro, F.*, Zé-Zé L.*, Alves, M. J.* and Alves de Matos, A. P.** \\ * Centro de Estudos de Vectores e Doenças Infecciosas/Instituto Nacional de Saúde Dr. Ricardo Jorge, \\ Avenida da Liberdade n. ${ }^{\circ}$ 5, 2965-575 Águas de Moura, PORTUGAL \\ ** Cooperativa de Ensino Superior - Egas Moniz, Campus Universitário, Quinta da Granja, 2829- 511 Monte \\ de Caparica, Almada, PORTUGAL
}

Email: fatima.amaro@insa.min-saude.pt

Phlebovirus genus is comprised by arthropod-borne viruses and is one of the five genera that compose Bunyaviridae family [1]. Viruses within this family mature intracellularly and their RNA replicates in the cytoplasm, while the viral proteins transit through the Endoplasmic Reticulum and Golgi apparatus [2]. Serological studies in human Portuguese populations have proven that Toscana virus, a neurotropic phlebovirus, is present in Portugal but there were no data about phleboviral activity in arthropod vectors [3]. In this context, and in order to detect sandflies naturally infected by phleboviruses, an entomological surveillance was conducted in Arrabida region, in the south of Portugal.

Sandflies were collected in 2007 and 2008 with cdc light-traps. Pools were separated according to sex, date and collection site and, after RNA extraction, a reverse transcription Polymerase chain reaction was performed with generic primers [4]. Positive pools were inoculated in new born mice brains or in Vero E6 cells. Isolation was achieved in one mice litter and flasks with Vero cells E6 were inoculated with their brain extracts. A second passage was done in Vero E6 cells. Electron microscopy was prepared as previously described [5].

Cells inoculated with brain extracts showed large proliferation of internal membranes producing large intracytoplasmic inclusions, and virions can were observed associated with the periphery of the inclusions (Fig.1).With subsequent passages the proliferation of the membranes became less marked and virons were observed at earlier times after inoculation, budding into the Golgi apparatus vacuoles (Fig 2). Virion morphology was consistent with the described morphology of bunyaviruses (Fig. 3).

Our results disclosed a modification of the virus morphogenesis in the course of adaptation of the virus to the Vero E6 cells. Extracellular and intracellular virions could be observed in all preparations confirming the production of virus particles by the infected cells.

Genomic sequences obtained (data not shown) confirmed that the virus, provisionally named Arrabida virus, is a phlebovirus closely related to phleboviruses within the Sandfly fever Naples virus complex.

This work was partially supported by an FCT project: New arboviruses isolated in Portugal. Risk assessment and public health application (PTDC/SAU-SAP/119199/2010). We wish to thank Lígia Chainho for the technical support.

[1] International Commitee on Taxonomy of Viruses (ICTV). Seventh Report of the International Commitee on Taxonomy of Viruses. Van Regenmortel M. H.V. et al. (eds). San Diego: Academic Press, 599-621, 2000.

[2] Nathenson N., and Gonzalez-Scarano F. Encyclopedia of virology Webster, R. G. \& Granoff, A. (eds). San Diego: Academic Press 185-192, 1994.

[3] Amaro F. et al., Epidemiology and Infection; 140, 1147-1150, 2012. doi: 10.1017/S0950268811001403. 
[4] Sánchez-Seco M. P. et al., Jounal of Medical Virology, 71, 140-9, 2003.

[5] Alves de Matos A.P. et al., Microscopy and Microanalysis. 17, 101-108, 2011.

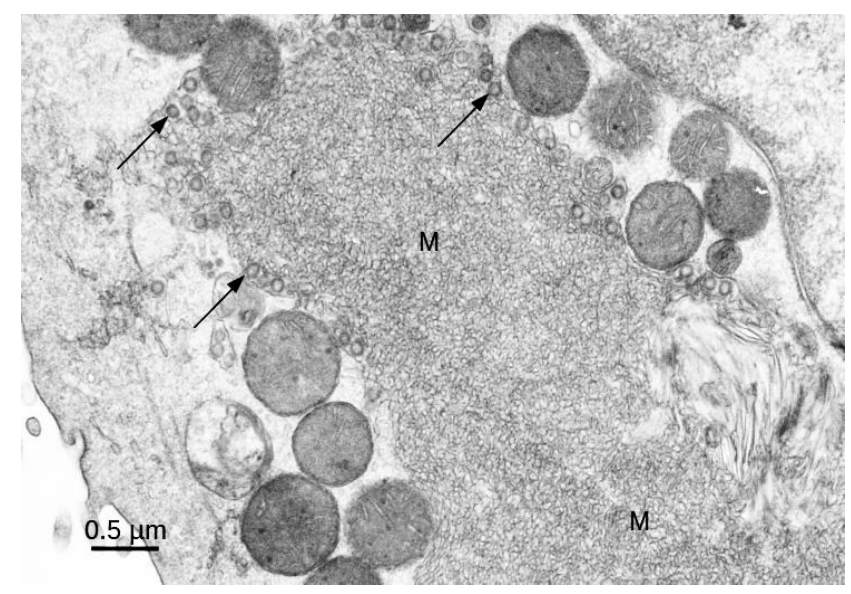

Fig 1- Vero E6 cells inoculated with brain extract of infected mice at 5 d.p.i.. Intracytoplasmic membranous inclusions (M) with virions (arrows) at the periphery.

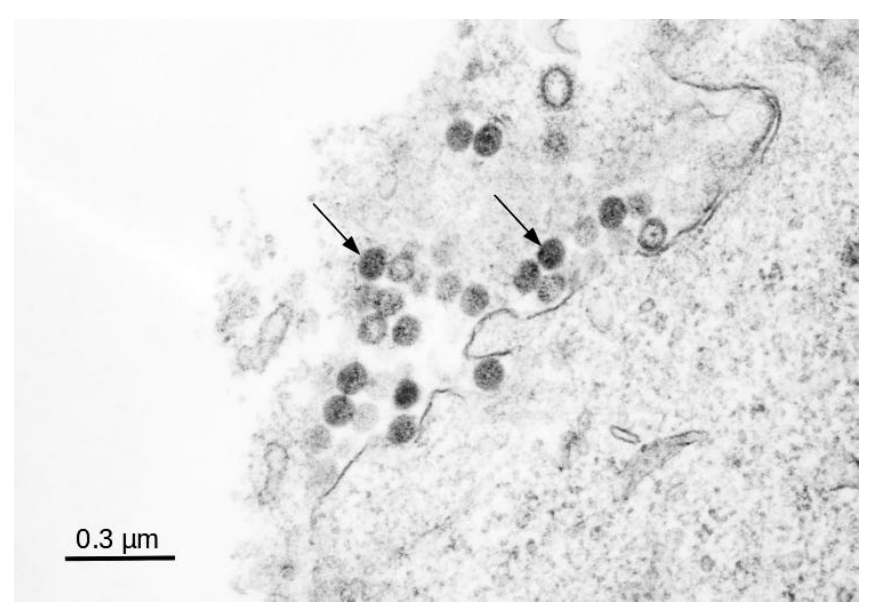

Fig. 2- Infected cell (2nd passage in Vero E6 Cells) at 2 d.p.i.. Virions (arrows) can be observed at the periphery of the cell.

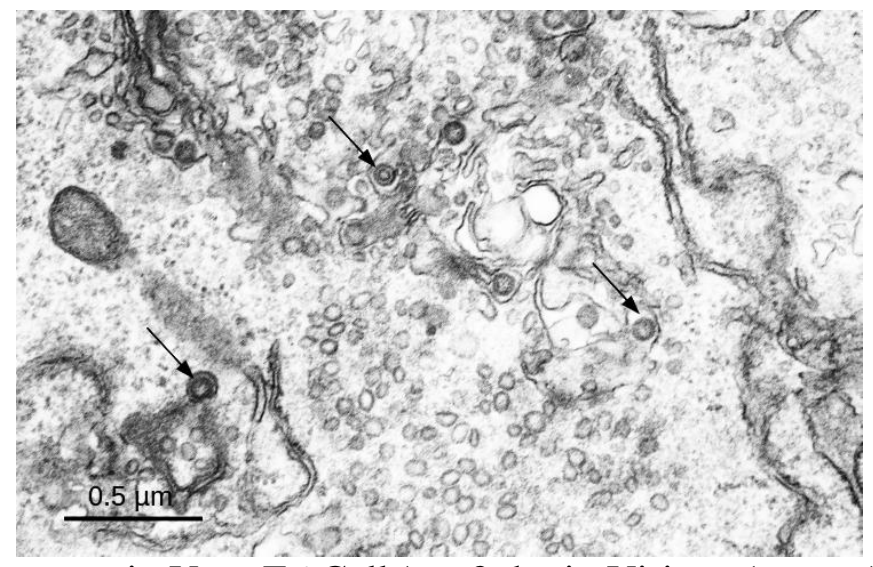

Fig.3- Infected cell (2nd passage in Vero E6 Cells) at 2 d.p.i.. Virions (arrows) budding into vacuoles of the Golgi apparatus. 